\title{
Association Study of a Monoamine Oxidase A Gene Promoter Polymorphism with Major Depressive Disorder and Antidepressant Response
}

\author{
Younger W-Y Yu', Shih-Jen Tsai ${ }^{2,3}$, Chen-Jee Hong ${ }^{2,3}$, Tai-Jui Chen*,4,5, Ming-Chao Chen ${ }^{6}$ and \\ Chih-Wei Yang \\ 'Yu's Psychiatric Clinic, Kaohsiung, Taiwan, ROC; '2Division of Psychiatry, School of Medicine, National Yang-Ming University, Taipei, Taiwan, ROC; \\ ${ }^{3}$ Department of Psychiatry, Veterans General Hospital-Taipei, Taipei, Taiwan, ROC; ${ }^{4}$ I-Shou University, Taiwan, ROC; ${ }^{5}$ E-DA Hospital, Kaohsiung, \\ Taiwan, ROC; ${ }^{6}$ Kai-Suan Psychiatric Hospital, Kaohsiung, Taiwan, ROC
}

\begin{abstract}
Monoamine oxidase $A(M A O A)$, a mitochondrial enzyme involved in the degradation of biogenic amines, may be implicated in the pathogenesis of major depressive disorder (MDD) and be related to the therapeutic effects of antidepressants. To elucidate a genetic predisposition of MDD, we studied a variable-number-tandem-repeat (VNTR) polymorphism in the promoter region of the MAOA gene in a Chinese population of 230 MDD patients and 217 controls. We also examined the association of this polymorphism and antidepressant therapeutic response in the MDD patients who underwent a 4-week fluoxetine treatment. Our results showed a significantly increased frequency of 4-repeat (4R) allele in MDD patients, especially in the female population. Furthermore, MDD female patients who were $3 R$ homozygotes had a significantly better response to 4-week fluoxetine treatment when compared to 4R carriers $(p=0.024)$, but there was a nonsignificant difference found in male patients $(p=0.081)$. Since the $4 R$ allele transcribed $2-10$ times more efficiently than those with $3 R$ allele, our findings suggest that the MAOA-uVNTR may be involved in the pathogenesis of MDD and the antidepressant therapeutic mechanisms in Chinese population, and that there may be a gender effect in this association.

Neuropsychopharmacology (2005) 30, 1719-1723. doi:I0.1038/sj.npp. I300785; published online I June 2005
\end{abstract}

Keywords: monoamine oxidase A; major depressive disorders; polymorphism; fluoxetine; treatment response

\section{INTRODUCTION}

Monoamine oxidase (MAO) is a mitochondrial outer membrane enzyme that degrades biogenic amines, including neurotransmitters such as norepinephrine, dopamine, and serotonin. Two forms of the enzyme, MAOA and $M A O B$, are found in the brain where under physiological conditions, norepinephrine and serotonin are preferentially metabolized by $M A O A$, and dopamine by both forms of the enzyme. Several lines of evidences indicate that MAOA may be involved in the pathogenesis of major depressive disorder (MDD). First, a significant elevation of $M A O A$ activity was found in the hypothalamic region of postmortem brain from suicide victims (Sherif et al, 1991). The difference was more pronounced in the subgroup of

\footnotetext{
*Correspondence: Dr T-J Chen, Department of Psychiatry, E-DA Hospital, No. I, Yi-Da Road, Jiau-Shu Tsuen, Yan-Chau Shiang, Kaohsiung Country, Taiwan, ROC, Tel: +886 7615001 I ext. 2650, Fax: +8867615 5352, E-mail: edI00239@edah.org.tw

Received I I October 2004; revised 3 I March 2005; accepted 26 April 2005

Online publication: 28 April 2005 at http://www.acnp.org/citations/ NPP042805040473/default.pdf
}

suicides with a record of depressive disorder. Second, $M A O$ inhibitors are effective drugs for the treatment of MDD. Third, since MAOA plays an important role in the metabolism and regulation of central serotonin and norepinephrine, which are important for the pathogenesis of MDD (Meltzer, 1989), it is likely that MAOA will play important role in the pathogenesis of MDD.

The MAOA gene has been mapped to the short arm of $\mathrm{X}$ chromosome (Lan et al, 1989), and a variable-numbertandem-repeat (VNTR) polymorphism in the promoter region of the MAOA gene (MAOA-uVNTR) has been identified ( $Z$ hu et al, 1992). The polymorphism, which is located $1.2 \mathrm{~kb}$ upstream of the MAOA coding sequences, consists of 30-bp repeated sequence present in 2, 3, 3.5, 4, or 5 repeats (R). This polymorphism is functional in that $3.5 \mathrm{R}$ or $4 \mathrm{R}$ transcribed 2-10 times more efficiently than those with 2, 3, or 5R (Sabol et al, 1998). Although there are several negative association studies between MAOA-uVNTR gene polymorphism and psychiatric phenotype (Furlong et al, 1999; Yoshida et al, 2002b; Kunugi et al, 1999; Syagailo et al, 2001; Garpenstrand et al, 2002) including an alcoholism study in Han Chinese males (Lu et al, 2002), previous reports demonstrated that this polymorphism may 
be associated with monoamine metabolite concentrations in the CSF of healthy volunteers (Jonsson et al, 2000) as well as psychiatric diseases such as panic disorder (Deckert et al, 1999), obsessive-compulsive disorder (Camarena et al, 1998), bipolar disorder (Preisig et al, 2000), Alzheimer's disease (Takehashi et al, 2002), and schizophrenia (Jonsson et al, 2003). Considering the possible role of $M A O A$ in the pathogenesis and therapeutic mechanisms, in this study, we investigated the possible association of the MAOA-uVNTR polymorphism with MDD and its antidepressant therapeutic response.

\section{METHODS}

The study was an add-on to another research project ( $\mathrm{Yu}$ et al, 2002) and was approved by the Institution Review Board of the Taipei Veteran General Hospital. A total of 230 psychiatric outpatients (age range 18-79 years) who met the Diagnostic and Statistical Manual of the American Psychiatric Association (DSM-IV) criteria for MDD were studied. The diagnoses were based on clinical interviews and bipolar depressive patients were excluded. Other inclusion criteria were (1) minimum baseline score of 18 on the 21 -item Hamilton Depression Rating Scale (HAM-D) (Hamilton, 1967) and (2) presence of depressive symptoms for at least 2 weeks before entry into the study, without antidepressant treatment (patients were fresh cases or had quit antidepressant treatment for more than 2 weeks). Exclusion criteria were additional diagnoses on Axis I (including substance abuse, generalized anxiety disorders, panic disorders, or obsessive compulsive disorders) of the DSMIV, personality disorders, pregnancy, attempted suicide, and major medical and/or neurological disorders. In total, 217 normal controls were recruited to be included for association study. Most of the normal controls were medical staff and they were each interviewed to rule out psychotic or mood disorders. Assessment of MDD patients was performed by a senior psychiatrist who is an expert in psychiatric rating (YWY). The sample consisted entirely of ethnic Han Chinese. Informed consents were obtained from all participants.

All 230 MDD patients took fluoxetine (range: $20-40 \mathrm{mg}$ / day; mean 25.7 (9.0) $\mathrm{mg} /$ day) and received treatment efficacy evaluation with the HAM-D before and after the 4-week fluoxetine treatment by one investigator (YWY) who was blinded to the patient's genotype. Therapeutic response was evaluated by the percentage score reduction in HAM-D scores ((baseline score-4-week score) $\times 100 /$ baseline score).

For MAOA-uVNTR genotyping, genomic DNA was extracted from EDTA-containing venous blood samples according to standard procedures. The fragments of MAOA$\mathrm{uVNTR}$ were amplified by polymerase chain reaction (PCR) using the forward primers of $5^{\prime}$-CCCAGGCTGCTCCA GAAAC- $3^{\prime}$ and a reverse primer of $5^{\prime}$-GGACCTGGG CAGTTGTGC-3'. Polymorphisms of MAOA-uVNTR were determined according to size, which was determined from $3 \%$ agarose-gel electrophoresis. The $30-\mathrm{bp}$ repeat polymorphism consists of five alleles: 2R: $179 \mathrm{bp}$; 3R: 209; 4R: $239 \mathrm{bp}$; 5R: $269 \mathrm{bp}$. Furthermore, a 3.5R allele has been observed containing three 30 -bp repeat and an additional 18-bp of an incomplete repeat unit (227 bp) (Samochowiec et al, 1999).

The categorical data were analyzed using the $\chi^{2}$ test or the Fisher's exact test, as appropriate. Differences in continuous variables were evaluated by Student's $t$-test (eg age difference between the MDD and control; comparison of the HAM-D change after fluoxetine treatment between two genotypic groups in male MDD) or one-way analysis of variance followed by the LSD multiple range tests for comparison among groups (eg comparison of the HAM-D change after fluoxetine treatment among three genotypic groups in female MDD). The criterion for significance was set at $p<0.050$ for all of the tests. Data are presented as mean (SD).

\section{RESULTS}

The frequency of 3.5R allele was zero; in addition, $2 \mathrm{R}$ and $5 \mathrm{R}$ allele were rare in this study. Thus, six individuals who carried $2 \mathrm{R}$ or $5 \mathrm{R}$ alleles (two MDD female patients carried one $2 \mathrm{R}$ allele, three normal female subects carried one $2 \mathrm{R}$ allele, and one normal female carried one $5 R$ ) were excluded in the following statistical analyses (thus, $\mathrm{MDD}=228$ and Normal =213). The age (MDD: $44.3 \pm 16.8$ years; controls: $41.8 \pm 10.3$ years) and sex (male/female: MDD: 95/133; controls: 110/103) distributions of the MDD patients and the control population were similar $(t=1.83, \mathrm{df}=439$, $p=0.063$ and $\chi^{2}=0.17, \mathrm{df}=1, p=0.20$, respectively). Owing to the fact that MAOA-uVNTR is on the $\mathrm{X}$ chromosome, we divided the total sample into two groups according to gender for analysis. The genotypes and allele distribution for the $M A O A$-uVNTR polymorphism for the MDD group and controls are shown in Table 1. The distributions of the MAOA genotypes for the female MDD cases and the female controls were each in Hardy-Weinberg equilibrium. Comparison of the $M A O A-\mathrm{uVNTR}$ polymorphism among female subjects showed significant differences for the genotype distribution between the two groups $\left(\chi^{2}=9.65, \mathrm{df}=2, p=0.008\right)$ with $4 \mathrm{R}$ carriers more common in the MDD group (Table 1). Using $3 \mathrm{R}$ homozygote as a baseline, the odds ratio for bearers of $4 \mathrm{R}$ allele was 2.39 (95\% CI 1.36-4.02). Analysis of the allele frequency demonstrated that $4 \mathrm{R}$ allele is more common in the MDD group when compared with the control group $\left(\chi^{2}=6.93\right.$, $\mathrm{df}=1, p=0.009$ ). Similarly, for males, $4 \mathrm{R}$ is more common in the MDD group compared with the control group $\left(\chi^{2}=6.27, \mathrm{df}=1, p=0.015\right.$ (Fisher's exact test) (Table 1$)$. Using 3R carriers as baseline, the odds ratio for bearers of $4 \mathrm{R}$ allele was 1.97 (95\% CI, 1.17-3.67). When comparing the total sample, including female and male subjects, there are still significant differences found for the allele distribution between the two groups $\left(\chi^{2}=12.48, \mathrm{df}=, p<0.001\right)$, with $4 \mathrm{R}$ carriers more common in the MDD group (Table 1). However, when considering the issue of X-inactivation at the MAOA locus, we used another alternative approach combining total males and only homozygous female subjects. The result also demonstrated significant differences between the two groups $\left(\chi^{2}=10.7,2, \mathrm{df}=1, p=0.001\right)$ with $4 \mathrm{R}$ carriers more common in the MDD group. In this study, the MAOA-uVNTR allele frequency is different for this Chinese population as compared to European- 
Table I Monoamine Oxidase A u-VNTR Allele and Genotype Distributions in Major Depressive Disorder (MDD) Patients and Normal Control Subjects

\begin{tabular}{|c|c|c|c|c|c|c|c|c|c|}
\hline & \multicolumn{9}{|c|}{ Allele frequencies } \\
\hline & \multicolumn{2}{|c|}{ Male } & $p$ & \multicolumn{2}{|c|}{ Female } & $p$ & \multicolumn{2}{|c|}{ Total } & $p$ \\
\hline MDD & 46 & 49 & 0.015 & 144 & 122 & 0.009 & $190(52.6 \%)$ & I7I (47.4\%) & $<0.001$ \\
\hline Control & 68 & 35 & & 145 & 75 & & $213(65.9 \%)$ & $110(34.1 \%)$ & \\
\hline & \multicolumn{9}{|c|}{ Genotype distribution (the female subjects) } \\
\hline & & & $3 R / 3 R$ & & $3 R / 4 R$ & & $4 R / 4 R$ & & $\boldsymbol{p}$ \\
\hline MDD & & & $34(25.6 \%)$ & & $76(57.1 \%)$ & & $23(17.3 \%)$ & & 0.008 \\
\hline Control & & & $49(44.5 \%)$ & & $47(42.7 \%)$ & & $14(12.7 \%)$ & & \\
\hline
\end{tabular}

American samples; however, our results are similar to the Japanese analog in which a high $3 \mathrm{R}$ allele frequency was demonstrated (3R allele frequency of control group: $65.9 \%$ in this study, $60 \%$ in the Japanese sample, and $25-38 \%$ in the European-American samples; $3 \mathrm{R}$ repeats allele frequency of major depressive group: $52.6 \%$ in this study, $58.4-59 \%$ in the Japanese sample, and $32 \%$ in the European-American samples) (Samochowiec et al, 2004; Schulze et al, 2000; Kunugi et al, 1999; Yoshida et al, 2002b).

Therapeutically, female patients bearing the $3 \mathrm{R}$ homozygous genotype had a significantly better response to antidepressants when compared with four repeats allele carriers, as evaluated on the HAM-D change after 4 weeks of fluoxetine treatment (3R/3R: $46.9 \pm 19.0 \%, \quad 3 \mathrm{R} / 4 \mathrm{R}$ : $37.3 \pm 19.8 \%, 4 \mathrm{R} / 4 \mathrm{R}: 34.1 \pm 19.4 \%$, respectively, $p=0.024)$. A multiple linear-regression analysis was performed using HAM-D change after fluoxetine treatment as the dependent variable, and age and MAOA-uVNTR genotype as the predictor variables. We included age as predictor variable because our earlier study found that age may have some effect on antidepressant therapeutic response (Yu et al, 2002). The results demonstrated that the MAOA-uVNTR genotype was a significant predictor of therapeutic response $\left(p=0.010 ; r^{2}=0.049\right)$. When comparing the 3R genotype with the $4 \mathrm{R}$ genotype group in male patients, there was nonsignificant difference found between $3 \mathrm{R}$ carriers and $4 \mathrm{R}$ carriers (3R: $39.1 \pm 21.0 \%, 4 \mathrm{R}: 31.0 \pm 23.6 \%$, respectively, $p=0.081)$ according to the change of HAM-D.

\section{DISCUSSION}

The present study investigated the association between the MAOA-uVNTR polymorphism and MDD in a Chinese population. We found a significant association, suggesting that the MAOA-uVNTR gene polymorphism may be involved in the MDD susceptibility for our sample population. This finding of an association between MAOA-uVNTR gene polymorphism and MDD is similar with Schulze's study which demonstrated increments in frequency of genotypes containing long alleles of MAOAuVNTR in female patients with recurrent major depression (Schulze et al, 2000). However, our findings contrast with previous reports that showed no association between the two (Kunugi et al, 1999; Syagailo et al, 2001). One possible explanation for these discrepant results is that the association between MDD status and carriage of the MAOAuVNTR polymorphism is in linkage disequilibrium with a functional polymorphism in or near the MAOA gene and the linkage disequilibrium is seen in some ethnic-groups, but not others. Another explanation is that out positive report is a false result due to population stratification. A potential limitation of this study is that case-control association studies can be influenced by undetected population stratification. We restricted the study cases to Han Chinese and focused on a polymorphism with demonstrated functional effects. Further studies with family-based designs or 'genomic control' analysis would be useful to confirm our findings. Finally, given the assumptions that MDD is probably multidetermined, if the MAOA-uVNTR polymorphism is an uncommon disease locus, or one of small effect, the power to detect the gene must consequently be reduced. The negative results in two previous reports could be due to lack of power because of the small sample size (Kunugi et al, 1999; Syagailo et al, 2001). Finally, the discrepancy may be due to the phenotypic heterogeneity of the MDD patients and the $M A O A-\mathrm{uVNTR}$ polymorphism may be just associated with a subgroup of MDD patients. Since the diagnosis of MDD is based on symptom-oriented criteria, selecting cases according to the diagnostic criteria does not assure an etiologically homogenous sample. For example, recent study had demonstrated that this $M A O A-\mathrm{uVNTR}$ polymorphism is related to impulsivity in male mood disorder patients (Huang et al, 2004).

Another finding of this study is that the female patients bearing the $M A O A-\mathrm{uVNTR} 3 \mathrm{R} / 3 \mathrm{R}$ genotype had a better response to 4 -week fluoxetine treatment than $4 \mathrm{R}$ allele carriers, and that a nonsignificant difference was found in male patients. When using another alternative approach 
combining males and only homozygous female subjects, the results showed that $3 \mathrm{R}$ allele carriers had a better response than $4 \mathrm{R}$ allele carriers ( $3 \mathrm{R}$ allele carriers: $42.4 \pm 20.5 \%, 4 \mathrm{R}$ allele carriers: $31.9 \pm 22.3 \%$, respectively, $p=0.003)$. Previously, Cusin et al (2002) reported no significant difference in response between $M A O A$-uVNTR genotypes in an Italy sample consisting of MDD or bipolar depressives treated with fluvoxamine or paroxetine for 6 weeks. Another study by Yoshida et al (2002b) reported no significant difference in antidepressant therapeutic response among the MAOAuVNTR genotype groups in 66 Japanese MDD patients treated with fluvoxamine for 6 weeks. The differences in the study design among their studies and ours included the therapeutic-response assessment (Montgomery and Asberg Depression Rating Scale was used in Yoshida's study), duration of treatment (4 weeks in our study vs 6 weeks in the other two studies), sample (bipolar depression was also included in Cusin's study), and choice of antidepressant (fluoxetine $v s$ fluvoxamine $v s$ paroxetine; pindolol augmentation was used in Cusin's study), which may account for the different results. Furthermore, in our study, it was determined that the MAOA-uVNTR polymorphism accounts for $4.9 \%$ of the variance in antidepressant response. Our study using a larger sample, in a homogenous population (Chinese MDD), single medication (fluoxetine), and single rater are more likely to detect this difference. Recent study by Peters et al, (2004) consisted of 96 subjects with unipolar major depression treated with fluoxetine with response variables assessed after a 12-week trial. The results implicated the MAOA gene in the specificity of response to fluoxetine, which was more compatible with the results of our study.

In this study, we demonstrated that higher expression allele (4R) of MAOA is associated with MDD susceptibility and poorer antidepressant response. Since serotonin is chiefly metabolized by MAOA, 4R carriers who have higher central MAOA activity are likely to have lower central serotonin levels than those of $3 \mathrm{R}$ carriers. Our findings are in line with the report of a significant elevation of central MAOA activity in depressive suicide victims (Sherif et al, 1991), and data from previous research suggesting that lower serotonergic function could be the pathogenesis of MDD and drugs that can increase synaptic serotonin levels have antidepressant effects (for a review, see Meltzer, 1989).

In our study, the associations of MAOA-uVNTR polymorphism with MDD susceptibility and fluoxetine treatment response were more prominent in the female sample. The study of Jonsson et al (2000) investigated the relationship between MAOA-uVNTR polymorphism and CSF concentrations of 5-hydroxyindoleacetic acid in healthy volunteers. They found that female subjects containing alleles with 3.5 and/or 4 repeats reported to induce a more effective MAO transcription (Deckert et al, 1999; Sabol et al, 1998), and display higher concentrations of 5-hydroxyindoleacetic acid, but this relevance did not occur in men. Also, several previous reports demonstrated a gender effect in the association between this polymorphism and mental problems (Deckert et al, 1999; Camarena et al, 1998; Preisig et al, 2000; Takehashi et al, 2002; Huang et al, 2004; Cohen et al, 2003; Eley et al, 2003; Jonsson et al, 2003; Williams et al, 2003). From these reports and our findings, it seems that there is a gender effect for $M A O A-\mathrm{uVNTR}$ polymorphism in psychiatric illness. Thus, it is important to take this gender effect into account while studying this polymorphism in mental diseases. The cause for the possible gender effect on this polymorphism is unknown. One possible explanation comes from the evidence that, in the transfected cell line, MAOA activity is reduced by estrogen treatment ( $\mathrm{Ma}$ et al, 1993). Furthermore, the $M A O A$ is on the $\mathrm{X}$ chromosome. Female subjects have two $\mathrm{X}$ chromosomes, but only one $\mathrm{X}$ chromosome is generally active (Willard, 2000). The $\mathrm{X}$ chromosome inactivation may have effect in the MAOA expression for the female MAOAuVNTR heterozygotes. Nevertheless, as many as $10-15 \%$ of chromosome in all cells, females are epigenetic mosaics (that is, some cells show one pattern, whereas other cells show a different pattern). A further consideration is that because inactivation takes place not at the point of conception, but rather later on near the time of implantation, there is a developmental window that allows for the development of differences in cellular chemistry between male and female subjects. Although inactivation is largely random in operation, it seems that environment can sometimes influence the inactivation pattern. These speculations may need further exploration. Finally, genetic influences are not determined by the genes inherited, but rather by the genes expressed. That is, the inherited DNA brings about its effects through the RNA that it influences in cells in which the gene is expressed. The relevance of this point is that gene expression is probably influenced by hormonal factors, among other features (Petronis, 2001).

Although this study produced several positive results, there were some limitations. First, the most obvious limitation of this study is that the duration for fluoxetine evaluation was 4 weeks, although there have also been some studies which used 4 weeks as the time point in short-term antidepressant treatment response evaluation (eg Minov et al, 2001; Serretti et al, 2001; Yoshida et al, 2002a). This is the minimal time expected for a treatment response and we may only identify rapid responders, not all responders, to fluoxetine treatment. Further study with a longer observation period is needed to identify all responders. Second, no correction was made in the obtained $p$-values for multiple testing in our study. After correction for multiple testing, the results of the association of MAOA-uVNTR polymorphism with fluoxetine treatment response stratified by gender were negative. Third, the $M A O A-\mathrm{uVNTR}$ polymorphism is proved to have putative functional nature, but we cannot exclude the effects of other polymorphisms for MAOA gene. Although strong linkage disequilibrium in the Chinese population was found between the MAOA-uVNTR and other MAOA gene polymorphism such as EcoRV polymorphism in exon 14 (Lu et al, 2002), using haplotypic analysis of multiple polymorphisms for $M A O A$ gene in future studies will be greatly helpful to better explore the role of $M A O A$ in depression.

\section{ACKNOWLEDGEMENTS}

This work was supported by grant NSC 90-2314-B-075-068 from the National Science Council, Taiwan, ROC and grant VGH89-328 from Veterans General Hospital-Taipei. 


\section{REFERENCES}

Camarena B, Cruz C, de la Fuente JR, Nicolini H (1998). A higher frequency of a low activity-related allele of the MAO-A gene in females with obsessive-compulsive disorder. Psychiatr Genet 8: 255-257.

Cohen IL, Liu X, Schutz C, White BN, Jenkins EC, Brown WT et al (2003). Association of autism severity with a monoamine oxidase A functional polymorphism. Clin Genet 64: 190-197.

Cusin C, Serretti A, Zanardi R, Lattuada E, Rossini D, Lilli R et al (2002). Influence of monoamine oxidase $A$ and serotonin receptor $2 \mathrm{~A}$ polymorphisms in SSRI antidepressant activity. Int J Neuropsychopharmacol 5: 27-35.

Deckert J, Catalano M, Syagailo YV, Bosi M, Okladnova O, Di Bella $\mathrm{D}$ et al (1999). Excess of high activity monoamine oxidase A gene promoter alleles in female patients with panic disorder. Hum Mol Genet 8: 621-624.

Eley TC, Tahir E, Angleitner A, Harriss K, McClay J, Plomin R et al (2003). Association analysis of MAOA and COMT with neuroticism assessed by peers. Am J Med Genet 120: 90-96.

Furlong RA, Ho L, Rubinsztein JS, Walsh C, Paykel ES, Rubinsztein DC (1999). Analysis of the monoamine oxidase A (MAOA) gene in bipolar affective disorder by association studies, metaanalyses, and sequencing of the promoter. Am J Med Genet 88: 398-406.

Garpenstrand H, Norton N, Damberg M, Rylander G, Forslund K, Mattila-Evenden $\mathrm{M}$ et al (2002). A regulatory monoamine oxidase a promoter polymorphism and personality traits. Neuropsychobiology 46: 190-193.

Hamilton M (1967). Development of a rating scale for primary depressive illness. Br J Soc Clin Psychol 6: 278-296.

Huang YY, Cate SP, Battistuzzi C, Oquendo MA, Brent D, Mann JJ (2004). An association between a functional polymorphism in the monoamine oxidase A gene promoter, impulsive traits and early abuse experiences. Neuropsychopharmacology 29: 1498-1505.

Jonsson EG, Norton N, Forslund K, Mattila-Evenden M, Rylander $\mathrm{G}$, Asberg $\mathrm{M}$ et al (2003). Association between a promoter variant in the monoamine oxidase A gene and schizophrenia. Schizophr Res 61: 31-37.

Jonsson EG, Norton N, Gustavsson JP, Oreland L, Owen MJ, Sedvall GC (2000). A promoter polymorphism in the monoamine oxidase A gene and its relationships to monoamine metabolite concentrations in CSF of healthy volunteers. J Psychiatr Res 34: 239-244.

Kunugi H, Ishida S, Kato T, Tatsumi M, Sakai T, Hattori M et al (1999). A functional polymorphism in the promoter region of monoamine oxidase-A gene and mood disorders. Mol Psychiatry 4: 393-395.

Lan NC, Heinzmann C, Gal A, Klisak I, Orth U, Lai E et al (1989). Human monoamine oxidase A and B genes map to Xp 11.23 and are deleted in a patient with Norrie disease. Genomics 4: 552-559.

Lu RB, Lee JF, Ko HC, Lin WW, Chen K, Shih JC (2002). No association of the MAOA gene with alcoholism among Han Chinese males in Taiwan. Prog Neuropsychopharmacol Biol Psychiatry 26: 457-461.

Ma ZQ, Bondiolotti GP, Olasmaa M, Violani E, Patrone C, Picotti GB et al (1993). Estrogen modulation of catecholamine synthesis and monoamine oxidase A activity in the human neuroblastoma cell line SK-ER3. J Steroid Biochem Mol Biol 47: 207-211.

Meltzer H (1989). Serotonergic dysfunction in depression. $\mathrm{Br} J$ Psychiatry Suppl 8: 25-31.

Minov C, Baghai TC, Schule C, Zwanzger P, Schwarz MJ, Zill P et al (2001). Serotonin-2A-receptor and -transporter polymorphisms: lack of association in patients with major depression. Neurosci Lett 303: 119-122.
Peters EJ, Slager SL, McGrath PJ, Knowles JA, Hamilton SP (2004). Investigation of serotonin-related genes in antidepressant response. Mol Psychiatry 9: 879-889.

Petronis A (2001). Human morbid genetics revisited: relevance of epigenetics. Trends Genet 17: 142-146.

Preisig M, Bellivier F, Fenton BT, Baud P, Berney A, Courtet P et al (2000). Association between bipolar disorder and monoamine oxidase A gene polymorphisms: results of a multicenter study. Am J Psychiatry 157: 948-955.

Sabol SZ, Hu S, Hamer D (1998). A functional polymorphism in the monoamine oxidase A gene promoter. Hum Genet 103: 273-279.

Samochowiec J, Lesch KP, Rottmann M, Smolka M, Syagailo YV, Okladnova O et al (1999). Association of a regulatory polymorphism in the promoter region of the monoamine oxidase A gene with antisocial alcoholism. Psychiatry Res 86: 67-72.

Samochowiec J, Syrek S, Michal P, Ryzewska-Wodecka A, Samochowiec A, Horodnicki J et al (2004). Polymorphisms in the serotonin transporter and monoamine oxidase A genes and their relationship to personality traits measured by the Temperament and Character Inventory and NEO FiveFactor Inventory in healthy volunteers. Neuropsychobiology 50: $174-181$.

Schulze TG, Muller DJ, Krauss H, Scherk H, Ohlraun S, Syagailo YV et al (2000). Association between a functional polymorphism in the monoamine oxidase A gene promoter and major depressive disorder. Am J Med Genet 96: 801-803.

Serretti A, Zanardi R, Cusin C, Rossini D, Lorenzi C, Smeraldi E (2001). Tryptophan hydroxylase gene associated with paroxetine antidepressant activity. Eur Neuropsychopharmacol 11: 375-380.

Sherif F, Marcusson J, Oreland L (1991). Brain gamma-aminobutyrate transaminase and monoamine oxidase activities in suicide victims. Eur Arch Psychiatry Clin Neurosci 241: 139-144.

Syagailo YV, Stober G, Grassle M, Reimer E, Knapp M, Jungkunz G et al (2001). Association analysis of the functional monoamine oxidase A gene promoter polymorphism in psychiatric disorders. Am J Med Genet 105: 168-171.

Takehashi M, Tanaka S, Masliah E, Ueda K (2002). Association of monoamine oxidase A gene polymorphism with Alzheimer's disease and Lewy body variant. Neurosci Lett 327: 79-82.

Willard HF (2000). The sex chromosomes and X chromosome inactivation. In: Scriver CR, Beaudet AL, Sly WS, Valle D, Childs $\mathrm{B}$, Vogelstein B (eds). The Metabolic and Molecular Bases of Inherited Disease. McGraw Hill: New York.

Williams RB, Marchuk DA, Gadde KM, Barefoot JC, Grichnik K, Helms MJ et al (2003). Serotonin-related gene polymorphisms and central nervous system serotonin function. Neuropsychopharmacology 28: 533-541.

Yoshida K, Ito K, Sato K, Takahashi H, Kamata M, Higuchi H et al (2002a). Influence of the serotonin transporter gene-linked polymorphic region on the antidepressant response to fluvoxamine in Japanese depressed patients. Prog Neuropsychopharmacol Biol Psychiatry 26: 383-386.

Yoshida K, Naito S, Takahashi H, Sato K, Ito K, Kamata M et al (2002b). Monoamine oxidase: a gene polymorphism, tryptophan hydroxylase gene polymorphism and antidepressant response to fluvoxamine in Japanese patients with major depressive disorder. Prog Neuropsychopharmacol Biol Psychiatry 26: 1279-1283.

Yu YWY, Tsai SJ, Chen TJ, Lin CH, Hong CJ (2002). Association study of the serotonin transporter promoter polymorphism and symptomatology and antidepressant response in major depressive disorders. Mol Psychiatry 7: 1115-1119.

Zhu QS, Grimsby J, Chen K, Shih JC (1992). Promoter organization and activity of human monoamine oxidase (MAO) A and B genes. J Neurosci 12: 4437-4446. 